\title{
New Construction and New Error Bounds for $(0,2,4)$ Lacunary Interpolation By Six Degree Spline
}

\author{
Karwan H. Jwamer \\ Ridha G. Karem \\ College of Education, University of Sulaimani, Iraq
}

Received on: 30 / 8 / 2009

Accepted on: 21 / 2 / 2010

\section{ABSTRACT}

The object of this paper obtains the existence, uniqueness and upper bounds for errors of six degree splines interpolating the lacunary data $(0,2,4)$. We also show that the changes of the boundary conditions and the class of spline functions has a main role in minimizing the upper bounds for error in lacunary interpolation problem. For this reason, in the construction of our spline function which interpolates the lacunary data $(0,2,4)$. We changed the boundary condition and the class of spline functions which are given by [4] from first derivative to third derivative and the class of spline function from $C^{2}[0,1]$ to $C^{4}[0,1]$.

Keywords: Lacunary interpolation, modulus of continuity, spline interpolations .

بناء جديد وحدود خطأ جديدة لإنداج فراغي (0 ، 2 ، 4) من الارجة السادة لدالة سبلاين

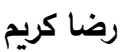

كروان جوامير

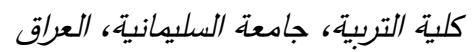

تاريخ استلام البحث: 2009/08/30

\section{الملخص}

إن هدف هذا البحث هو الحصول على الوجود، والوحدانية، والحدود العليا، من الأخطاء في الدرجة السادسة لدالة

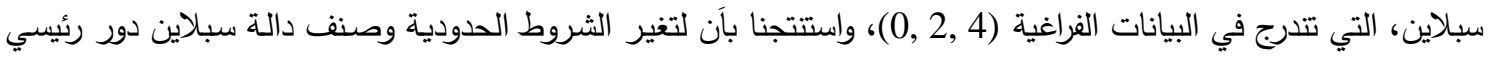

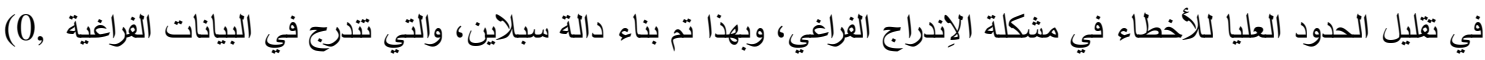

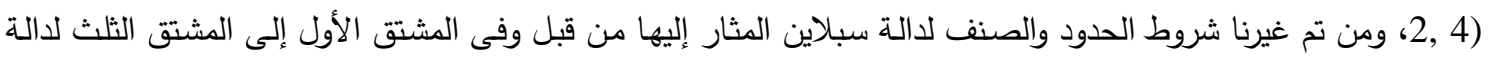

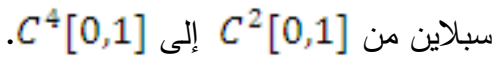

\section{Introduction:}

$$
\text { الكلمات المفتاحية: اندراج فراغي، معامل الاستمرارية ، اندراج سبلاين. }
$$

During the middle of twentieth century of spline has received considerable. According to Schoenberg [7], the interest in spline functions is due to the fact that spline functions are a good tool for the numerical approximation of functions on one hand and that they suggest new, challenging and rewarding problems on the other hand Piecewise Linear functions, as well as step functions, have long been an important theoretical and Practical tools for approximation of functions. A notable exception was the work done by actuarial mathematicians which is called 'osulatory interpolation' that began soon after Hermit's work on interpolation. To get more information about a spline function, one can see it in Alberg, Nilson and Walsh's theory [1]. In the last four decades Birkhoff [2] has renewed this theory on lacunary interpolation or interpolation by splines. Lacunary interpolation by spline appears whenever observation gives scattered or irregular information about a function and it's derivatives. The data in the problem of lacunary interpolation has also values of the functions and of it's derivatives but without Hermite condition that only consecutive derivatives is used at each nodes. Mathematically in the problem of interpolating gives us this data $a_{i, j}$ by a polynomial $p_{n}(x)$ of degree at most $\mathrm{n}$ satisfying:

$p_{n}^{(j)}\left(x_{i}\right)=a_{i, j}, i=1,2, \ldots, n_{j} j=0,1,2, \ldots, m$ 
We have Hermite interpolation if for each $i$, the order $j$ of derivatives in (1) from unbroken Sequence. If some of the sequences are broken, we have lacunary interpolation

\section{Descriptions of the Method: $[3,6]$}

In this section, we present six degree splines interpolation for one dimensional and sufficiently smooth function $f(x)$ defined on $\mathrm{I}=[0,1]$, and $\Delta_{x}: 0=x_{0}<x_{2}<\cdots<x_{2 m}=1$ denote the uniform partition of I with knots $x_{i}=i h, h=\frac{1}{2 m}, t_{2 i}=x_{2 i}+\frac{2}{3} h$ where $i=0,2, \ldots, 2 m$ and $n=2 m+1$. We define the class of spline function $S_{n, 6}^{4}$ where $S_{n, 6}^{4}$ denotes the class of all splines of degree six which belongs to $C^{4}[0,1]$, and $\mathrm{n}$ is the number of knots, as follow:

Any element $S_{\Delta}(x) \in S_{n, 6}^{4}$ if the following two conditions are satisfied:

(i) $S_{\triangle}(x) \in C^{4}[0,1]$

(ii) $S_{\Delta}(x)$ is apolynomial of degree six in each $\left[x_{2 i}, x_{2 i+2}\right]$,

$$
i=0,1, \ldots, m-1
$$

\section{Construction of the Spline Function}

If $\mathrm{P}(\mathrm{x})$ is a polynomial of degree six on $[0,1]$, then we have $[3,6]$

$$
\begin{aligned}
P(x)= & P(0) A_{0}(x)+P\left(\frac{1}{3}\right) A_{1}(x)+P(1) A_{2}(\mathrm{x}) \oplus \mathrm{P}^{\mathrm{m}}\left(\frac{1}{3}\right) A_{3}(\mathrm{x})+\mathrm{P}^{\mathrm{m}}(0) A_{4}(x)+P^{m \prime}(1) A_{5}(x) \\
& +P^{(4)}\left(\frac{1}{3}\right) A_{6}(x) .
\end{aligned}
$$

where

$$
\begin{aligned}
& A_{0}(x)=\frac{1}{61}\left(243 x^{6}-972 x^{5}+1215 x^{4}-495 x^{2}-52 x+61\right) \\
& A_{1}(x)=\frac{1}{122}\left(-729 x^{6}+2916 x^{5}-3645 x^{4}+1485 x^{2}-27 x\right) \\
& A_{2}(x)=\frac{1}{122}\left(243 x^{6}-972 x^{5}+1215 x^{4}-495 x^{2}+131 x\right) \\
& A_{3}(x)=\frac{1}{122}\left(-81 x^{6}+324 x^{5}-405 x^{4}+226 x^{2}-64 x\right) \\
& A_{4}(x)=\frac{1}{32940}\left(-2835 x^{6}+9693 x^{5}-11430 x^{4}+5490 x^{3}-935 x^{2}+17 x\right) \\
& A_{5}(x)=\frac{1}{32940}\left(405 x^{6}+27 x^{5}-720 x^{4}+395 x^{2}-107 x\right) \\
& A_{6}(x)=\frac{1}{65880}\left(-3645 x^{6}+11286 x^{5}-9990 x^{4}+3155 x^{2}-806 x\right)
\end{aligned}
$$

\section{In the subsequent section we need the following values:}

For $f \in C^{5}[0,1]$ we have the following expansions $[3,6]$

$$
\begin{aligned}
& f\left(x_{2 i+2}\right)=f\left(x_{2 i}\right)+ 2 h f^{\prime}\left(x_{2 i}\right)+2 h^{2} f^{\prime \prime}\left(x_{2 i}\right)+\frac{4}{3} h^{3} f^{\prime \prime}\left(x_{2 i}\right)+\frac{2}{3} h^{4} f^{(4)}\left(x_{2 i}\right)+ \\
& \frac{4}{15} h^{5} f^{(5)}\left(\emptyset_{1,2 i}\right), x_{2 i}<\emptyset_{1,2 i}<x_{2 i+2} \\
& f\left(x_{2 i-2}\right)=f\left(x_{2 i}\right)- 2 h f^{\prime}\left(x_{2 i}\right)+2 h^{2} f^{\prime \prime}\left(x_{2 i}\right)-\frac{4}{3} h^{3} f^{\prime \prime}\left(x_{2 i}\right)+\frac{2}{3} h^{4} f^{(4)}\left(x_{2 i}\right)- \\
& \frac{4}{15} h^{5} f^{(5)}\left(\emptyset_{2,2 i}\right), x_{2 i-2}<\emptyset_{2,2 i}<x_{2 i} \\
& f\left(t_{2 i}\right)=f\left(x_{2 i}\right)+\frac{2}{3} h f^{\prime}\left(x_{2 i}\right)+\frac{2}{9} h^{2} f^{\prime \prime}\left(x_{2 i}\right)+\frac{4}{81} h^{3} f^{\prime \prime}\left(x_{2 i}\right)+\frac{2}{243} h^{4} f^{(4)}\left(x_{2 i}\right)+ \\
& \frac{4}{3645} h^{5} f^{(5)}\left(\emptyset_{3,2 i}\right), x_{2 i}<\emptyset_{3,2 i}<t_{2 i}
\end{aligned}
$$




$$
\begin{aligned}
& f\left(t_{2 i-2}\right)=f\left(x_{2 i}\right)-\frac{4}{3} h f^{\prime}\left(x_{2 i}\right)+\frac{8}{9} h^{2} f^{\prime \prime}\left(x_{2 i}\right)-\frac{32}{81} h^{2} f^{\prime \prime}\left(x_{2 i}\right)+\frac{32}{243} h^{4} f^{(4)}\left(x_{2 i}\right)- \\
& \frac{128}{3645} h^{5} f^{(5)}\left(\emptyset_{4,2 i}\right), t_{2 i-2}<\emptyset_{4,2 i}<x_{2 i} \\
& f^{\prime}\left(t_{2 i}\right)=f^{\prime}\left(x_{2 i}\right)+\frac{2}{3} h f^{\prime \prime}\left(x_{2 i}\right)+\frac{2}{9} h^{2} f^{\prime \prime \prime}\left(x_{2 i}\right)+\frac{4}{81} h^{3} f^{(4)}\left(x_{2 i}\right)+\frac{2}{243} h^{4} f^{(5)}\left(\emptyset_{5,2 i}\right) \text {, } \\
& x_{2 i}<\emptyset_{5,2 i}<t_{2 i} \\
& f^{\prime \prime}\left(t_{2 i}\right)=f^{\prime \prime}\left(x_{2 i}\right)+\frac{2}{3} h f^{\prime \prime}\left(x_{2 i}\right)+\frac{2}{9} h^{2} f^{(4)}\left(x_{2 i}\right)+\frac{4}{81} h^{3} f^{(5)}\left(\emptyset_{6,2 i}\right) \text {, } \\
& x_{2 i}<\emptyset_{6,2 i}<t_{2 i} \\
& f^{\prime \prime}\left(t_{2 i-2}\right)=f^{\prime \prime}\left(x_{2 i}\right)-\frac{4}{3} h f^{\prime \prime}\left(x_{2 i}\right)+\frac{8}{9} h^{2} f^{(4)}\left(x_{2 i}\right)-\frac{32}{81} h^{3} f^{(5)}\left(\emptyset_{7,2 i}\right) \text {, } \\
& t_{2 i-2}<\emptyset_{7,2 i}<x_{2 i} \\
& f^{\prime \prime}\left(x_{2 i+2}\right)=f^{\prime \prime}\left(x_{2 i}\right)+2 h f^{(4)}\left(x_{2 i}\right)+2 h^{2} f^{(5)}\left(\emptyset_{8,2 i}\right), \quad x_{2 i}<\emptyset_{8,2 i}<x_{2 i+2} \\
& f^{\prime \prime}\left(x_{2 i-2}\right)=f^{\prime \prime}\left(x_{2 i}\right)-2 h f^{(4)}\left(x_{2 i}\right)+2 h^{2} f^{(5)}\left(\emptyset_{9,2 i}\right) \text {, } \\
& f^{(4)}\left(t_{2 i}\right)=f^{(4)}\left(x_{2 i}\right)+\frac{2}{3} h f^{(5)}\left(\emptyset_{10,2 i}\right) \text {, } \\
& x_{2 i-2}<\emptyset_{9,2 i}<x_{2 i} \\
& x_{2 i}<\emptyset_{10,2 i}<t_{2 i} \\
& f^{(4)}\left(t_{2 i-2}\right)=f^{(4)}\left(x_{2 i}\right)-\frac{4}{3} h f^{(5)}\left(\varnothing_{11,2 i}\right) \\
& t_{2 i-2}<\emptyset_{11_{0} 2 i}<x_{2 i} \\
& \left.f^{\mathrm{m}}\left(t_{2 i}\right)=f^{\mathrm{m}}\left(x_{2 i}\right)+\frac{2}{3} h f^{(4)}\left(x_{2 i}\right)+\frac{2}{9} h^{2} f^{(5)}\left(\emptyset_{12,2 i}\right) \quad x_{2 i}<\emptyset_{12,2 i}<t_{2 i}\right]
\end{aligned}
$$

\section{Existence and Uniqueness:}

In this section we prove the following theorem about the existence and uniqueness of the spline function $S_{n, 6}^{4}$

\section{Theorem 1:}

Given arbitrary numbers $f\left(x_{2 i}\right), f^{(r)}\left(t_{2 i}\right), i=0,1, \ldots, m-1 ; r=0,2,4$ and $f^{m}\left(x_{0}\right)_{n} f^{\mathrm{m}}\left(x_{2 m}\right)$ there exists a unique spline $S_{n}(x) \in S_{n, 6}^{4}$ such that

$$
\left.\begin{array}{l}
S_{n}\left(x_{2 i}\right)=f\left(x_{2 i}\right), i=0,1, \ldots, m \\
S_{n}^{(r)}\left(t_{2 i}\right)=f^{(r)}\left(t_{2 i}\right), i=0,1, \ldots, m-1 ; r=0,2,4 \\
S_{n}^{\mathrm{m}}\left(x_{0}\right)=f^{m}\left(x_{0}\right), S_{n}^{\mathrm{m}}\left(x_{2 m}\right)=f^{\omega}\left(x_{2 m}\right)
\end{array}\right\}
$$

\section{Proof:}

The proof depends on the following representation of $S_{n}(x)$ for $2 i h \leq x \leq(2 i+2) h$, $i=0,1, \ldots, m-1$. We have

$$
\begin{aligned}
S_{n}(x)= & f\left(x_{2 i}\right) A_{0}\left(\frac{x-2 i h}{2 h}\right)+f\left(t_{2 i}\right) A_{1}\left(\frac{x-2 i h}{2 h}\right)+f\left(x_{2 i+2}\right) A_{2}\left(\frac{x-2 i h}{2 h}\right)+4 h^{2} f^{\omega}\left(t_{2 i}\right) A_{3}\left(\frac{x-2 i h}{2 h}\right)+ \\
& 8 h^{3} S_{n}^{\mathrm{m}}\left(x_{2 i}\right) A_{4}\left(\frac{x-2 i h}{2 h}\right)+8 h^{3} S_{n}^{\omega}\left(x_{2 i}\right) A_{5}\left(\frac{x-2 i h}{2 h}\right)+16 h^{4} f^{(4)}\left(t_{2 i}\right) A_{6}\left(\frac{x-2 i h}{2 h}\right) . \quad \text { (7) }
\end{aligned}
$$

On using eq.(7) and the conditions

$S_{n}^{\mathrm{m}}(0)=f^{\mathrm{m}}(0)_{n} S_{n}^{\mathrm{m}}(1)=f^{\mathrm{m}}(1)$.

We see that $S_{n}(x)$ as given by (7) satisfies (2) and is sextic in $\left[x_{2 i}, x_{2 i+2}\right]$, i=0, $1, \ldots, \mathrm{m}-1$. We also need to show that whether it is possible to determine $S_{n,} i=1,2, \ldots, m-1$ uniquely. For this purpose we use the fact that 
$S_{n}^{(4)}\left(x_{2 i+}\right)=S_{n i}^{(4)}\left(x_{2 i-}\right), i=1,2, \ldots, m-1$; where $S_{n}^{(4)}\left(x_{2 i+}\right)=\lim _{x \rightarrow x_{n i}+} S_{n}^{(4)}(x)$ and $S_{n}^{(4)}\left(x_{2 i-}\right)=\lim _{x \rightarrow x_{n i}}-S_{n}^{(4)}(x)$, with the help of (7) and (8) reduced to

$$
\begin{gathered}
-2 h^{3} S_{n}\left(x_{2 i-2}\right)+\frac{376}{61} h^{3} S_{n}\left(x_{2 i}\right)+\frac{16}{61} h^{3} S_{n}^{\omega}\left(x_{2 i+2}\right)=\frac{3645}{122} f\left(x_{2 i}\right)-\frac{10935}{244} f\left(t_{2 i}\right)+ \\
\frac{3645}{244} f\left(x_{2 i+2}\right)-\frac{1215}{61} h^{2} f^{ \pm}\left(t_{2 i}\right)+3 h^{4} f^{(4)}\left(t_{2 i-2}\right)-\frac{222}{61} h^{4} f^{(4)}\left(t_{2 i}\right)_{x}
\end{gathered}
$$

$i=1,2, \ldots, m-1$. Equation (9) is a strictly tri-diagonal dominant system which has a unique solution (see [5] ). Thus $S_{n}^{\mathrm{m}}\left(x_{2 i}\right), i=1,2_{y} \ldots, m-1$ can be obtained uniquely by the system (9) which established Theorem 1 .

\section{Error bounds:} following:

In this section, the upper bounds for errors studied first helping results of the

\section{Lemma 1:}

Let us write $B_{2 i}=\left|S_{n}^{m}\left(x_{2 i}\right)-f^{m}\left(x_{2 i}\right)\right|$, then for $f \in C^{5}[0,1]$, we have $\max B_{2 i} \leq \frac{487}{270} h^{2} w\left(f^{(5)} ; \frac{1}{m}\right)$, for $i=1,2, \ldots, m-1$

where $w\left(f^{(5)} ; \frac{1}{m}\right)=\max \left\{\left|f^{(5)}(x)-f^{(5)}(y)\right| ;|x-y| \leq \frac{1}{m}\right\}$.

\section{Proof:}

From (8) we have

$$
\begin{aligned}
& -2 h^{3}\left(s_{n}^{m}\left(x_{2 i-2}\right)-f^{m}\left(x_{2 i-2}\right)\right)+\frac{376}{61} h^{3}\left(s_{n}^{m}\left(x_{2 i}\right)-f^{m}\left(x_{2 i}\right)\right)+ \\
& \frac{16}{61} h^{3}\left(S_{n}^{\omega}\left(x_{2 i+2}\right)-f^{\omega}\left(x_{2 i+2}\right)\right)=\frac{3645}{122} f\left(x_{2 i}\right)-\frac{10935}{244} f\left(t_{2 i}\right)+\frac{3645}{244} f\left(x_{2 i+2}\right)-\frac{1215}{61} h^{2} f^{\omega}\left(t_{2 i}\right) \\
& +3 h^{4} f^{(4)}\left(t_{2 i-2}\right)-\frac{222}{61} h^{4} f^{(4)}\left(t_{2 i}\right)+2 h^{3} f^{m}\left(x_{2 i-2}\right)-\frac{376}{61} h^{3} f^{m}\left(x_{2 i}\right)-\frac{16}{61} h^{3} f^{m}\left(x_{2 i+2}\right) \\
& =\frac{-3}{61} h^{5} f^{(5)}\left(\emptyset_{3,2 i}\right)+\frac{243}{61} h^{5} f^{(5)}\left(\emptyset_{1,2 i}\right)-\frac{60}{61} h^{5} f^{(5)}\left(\emptyset_{6,2 i}\right)-4 h^{5} f^{(5)}\left(\emptyset_{11,2 i}\right) \\
& \frac{148}{61} h^{5} f^{(5)}\left(\emptyset_{10,2 i}\right)+4 h^{5} f^{(5)}\left(\emptyset_{9,2 i}\right)-\frac{32}{61} h^{5} f^{(5)}\left(\emptyset_{8,2 i}\right)=\frac{487}{270} h^{2} \alpha_{1} w\left(f^{(5)} ; \frac{1}{m}\right), \quad\left|\alpha_{1}\right| \leq 1
\end{aligned}
$$

The result (10) follows on using the property of diagonal dominant [5].

\section{Lemma 2:}

Let $f \in C^{5}[0,1]$ then

$$
\begin{aligned}
& \left|S_{n}^{(4)}\left(x_{2 i+}\right)-f^{(4)}\left(x_{2 i}\right)\right| \leq \frac{730}{61} h w\left(f^{(5)} ; \frac{1}{m}\right), \\
& \left|S_{n}^{(4)}\left(x_{2 i}\right)-f^{(4)}\left(x_{2 i}\right)\right| \leq \frac{1514}{135} h w\left(f^{(5)} ; \frac{1}{m}\right), \\
& \left|S_{n}^{v}\left(t_{2 i}\right)-f^{v}\left(t_{2 i}\right)\right| \leq \frac{8471}{74115} h^{4} w\left(f^{(5)} ; \frac{1}{m}\right), \\
& \left|S_{n}^{\mathrm{w}}\left(t_{2 i}\right)-f^{\mathrm{w}}\left(t_{2 i}\right)\right| \leq \frac{28693}{16470} h^{2} w\left(f^{(5)} ; \frac{1}{m}\right),
\end{aligned}
$$


and

$$
\left|S_{n}^{(6)}\left(t_{2 i}\right)\right| \leq \frac{2947}{122} h^{-1} w\left(f^{(5)}, \frac{1}{m}\right),
$$

\section{Proof:}

From (7) we have

$$
\begin{array}{r}
h^{4} S_{n}^{(4)}\left(x_{2 i+}\right)=\frac{3645}{122} f\left(x_{2 i}\right)-\frac{10935}{244} f\left(t_{2 i}\right)+\frac{3645}{244} f\left(x_{2 i+2}\right)-\frac{1215}{61} h^{2} f^{\omega}\left(t_{2 i}\right)-\frac{254}{61} h^{3} S_{n}^{m}\left(x_{2 i}\right)- \\
\frac{16}{61} h^{3} S_{n}^{\omega}\left(x_{2 i+2}\right)-\frac{222}{61} h^{4} f^{(4)}\left(t_{2 i}\right)
\end{array}
$$

Hence

$$
\begin{aligned}
& h^{4}\left(s_{n}^{(4)}\left(x_{2 i+}\right)-f^{(4)}\left(x_{2 i}\right)\right)=\frac{-3}{61} h^{5} f^{(5)}\left(\emptyset_{3,2 i}\right)+\frac{243}{61} h^{5} f^{(5)}\left(\emptyset_{1,2 i}\right)-\frac{60}{61} h^{5} f^{(5)}\left(\emptyset_{6,2 i}\right)- \\
& \frac{148}{61} h^{5} f^{(5)}\left(\emptyset_{10,2 i}\right)-\frac{32}{61} h^{5} f^{(5)}\left(\emptyset_{8,2 i}\right)-\frac{254}{61} h^{3}\left(s_{n}^{\omega}\left(x_{2 i}\right)-f^{\omega}\left(x_{2 i}\right)\right)-\frac{16}{61} h^{3}\left(S_{n}^{\omega}\left(x_{2 i+2}\right)-\right. \\
& \left.f^{m}\left(x_{2 i+2}\right)\right)=\frac{243}{61} h^{5} \alpha_{2} w\left(f^{(5)} ; \frac{1}{m}\right)-\frac{254}{61} h^{3}\left(S_{n}^{m}\left(x_{2 i}\right)-f^{m}\left(x_{2 i}\right)\right)-\frac{16}{61} h^{3}\left(S_{n}^{ \pm}\left(x_{2 i+2}\right)-\right. \\
& \left.f^{m}\left(x_{2 i+2}\right)\right),\left|\alpha_{2}\right| \leq 1
\end{aligned}
$$

By using (10), we get (11). The proofs of (12)-(15) are similar, and we only mention that

$$
\begin{aligned}
& h^{4}\left(S_{n}^{(4)}\left(x_{2 i-}\right)=-2 h^{3} S_{n}^{m}\left(x_{2 i-2}\right)+2 h^{3} S_{n}^{\omega}\left(x_{2 i}\right)-3 h^{4} f^{(4)}\left(t_{2 i-2}\right)\right. \text {, } \\
& h^{3} S_{n}^{ \pm}\left(t_{2 i}\right)=\frac{540}{61} f\left(x_{2 i}\right)-\frac{810}{61} f\left(t_{2 i}\right)+\frac{270}{61} f\left(x_{2 i+2}\right)-\frac{360}{61} h^{2} f^{ \pm}\left(t_{2 i}\right)-\frac{12}{61} h^{3} S_{n}^{\omega}\left(x_{2 i}\right)- \\
& \frac{7}{61} h^{3} S_{n}^{m}\left(x_{2 i+2}\right)-\frac{116}{183} h^{4} f^{(4)}\left(t_{2 i}\right)_{x} \\
& h S_{n}^{\circ}\left(t_{2 i}\right)=-\frac{128}{61} f\left(x_{2 i}\right)+\frac{585}{244} f\left(t_{2 i}\right)-\frac{73}{244} f\left(x_{2 i+2}\right)+\frac{134}{183} h^{2} f^{\prime \prime}\left(t_{2 i}\right)+\frac{424}{8235} h^{4} f^{(4)}\left(t_{2 i}\right)+ \\
& \frac{176}{24705} h^{3} S_{n}^{m}\left(x_{2 i}\right)+\frac{184}{24705} h^{3} S_{n}^{m}\left(x_{2 i+2}\right)
\end{aligned}
$$

and

$$
\begin{aligned}
h^{6} S_{n}^{(6)}\left(t_{2 i}\right)=\frac{10935}{244} f\left(x_{2 i}\right)- & \frac{32805}{488} f\left(t_{2 i}\right)+\frac{10935}{488} f\left(x_{2 i+2}\right)-\frac{3645}{122} h^{2} f^{\square}\left(t_{2 i}\right)- \\
& -\frac{945}{122} h^{3} S_{n}^{\omega}\left(x_{2 i}\right)+\frac{135}{122} h^{3} S_{n}^{\omega}\left(x_{2 i+2}\right)-\frac{1215}{122} h^{4} f^{(4)}\left(t_{2 i}\right)
\end{aligned}
$$

\section{Theorem 2:}

Let $f \in C^{5}[0,1]$ and $S_{n}(x) \in S_{n, 6}^{4}$ be a unique spline satisfying the conditions of Theorem 1 , then

$\left\|S_{n}^{(r)}(x)-f^{(r)}(x)\right\| \leq \frac{145491106}{2371680} m^{r-5} w\left(f^{(5)} ; \frac{1}{m}\right)+2 m^{r-5}\left\|f^{(5)}\right\|, \mathrm{r}=0,1,2,3,4$

where $\left\|f^{(5)}\right\|=\max \left\{\left|f^{(5)}(x)\right| ; 0 \leq x \leq 1\right\}$.

\section{Proof:}

For $0 \leq y \leq 1_{y}$ we obtain

$$
A_{0}(y)+A_{1}(y)+A_{2}(y)=1 \text {. }
$$


Let $x_{2 i} \leq x \leq x_{2 i+2}$. On using (16) and (7), we obtain

$$
\begin{array}{r}
S_{n}^{(4)}(x)-f^{(4)}(x)=\left(S_{n}^{(4)}\left(x_{2 i+}\right)-f^{(4)}(x)\right) A_{0}\left(\frac{x-2 i h}{2 h}\right)+\left(S_{n}^{(4)}\left(t_{2 i}\right)-f^{(4)}(x)\right) A_{1}\left(\frac{x-2 i h}{2 h}\right)+ \\
\left(S_{n}^{(4)}\left(x_{2 i}\right)-f^{(4)}(x)\right) A_{2}\left(\frac{x-2 i h}{2 h}\right)+4 h^{2} S_{n}^{(6)}\left(t_{2 i}\right) A_{3}\left(\frac{x-2 i h}{2 h}\right)=E_{1}+E_{2}+E_{3}+E_{4}
\end{array}
$$

From (3) it follows that:

$\left|A_{0}(x)\right| \leq 1,\left|A_{1}(x)\right| \leq 1,\left|A_{2}(x)\right| \leq 1$ and $\left|A_{3}(x)\right| \leq 1$ on $0 \leq x \leq 1$

Since $f^{(4)}(x)=f^{(4)}\left(x_{2 i}\right)+\left(x-x_{2 i}\right) f^{(5)}\left(\emptyset_{13,2 i}\right) \quad x_{2 i} \leq \emptyset_{13,2 i} \leq x$

$E_{1}=\left(S_{n}^{(4)}\left(x_{2 i+}\right)-f^{(4)}(x)\right) A_{0}\left(\frac{x-2 i h}{2 h}\right)=$

$A_{0}\left(\frac{x-2 i h}{2 h}\right)\left(S_{n}^{(4)}\left(x_{2 i+}\right)-f^{(4)}\left(x_{2 i}\right)-\left(x-x_{2 i}\right) f^{(5)}\left(\emptyset_{13,2 i}\right)\right)$

On using (2) and $\left|x-x_{2 i}\right| \leq 2 h$ we obtain

$$
\left|E_{1}\right| \leq \frac{730}{61} h w\left(f^{(5)} ; \frac{1}{m}\right)+2 h\left\|f^{(5)}\right\|_{60}
$$

Similarly,

$$
\left|E_{3}\right| \leq \frac{1514}{135} h w\left(f^{(5)} ; \frac{1}{m}\right)+2 h\left\|f^{(5)}\right\|_{5 a^{3}}
$$

and

$$
\begin{aligned}
& E_{2}=\left(S_{n}^{(4)}\left(t_{2 i}\right)-f^{(4)}(x)\right) A_{1}\left(\frac{x-2 i h}{2 h}\right)=A_{1}\left(\frac{x-2 i h}{2 h}\right)\left(S_{n}^{(4)}\left(t_{2 i}\right)+f^{(4)}\left(t_{2 i}\right)-f^{(4)}\left(t_{2 i}\right)-\right. \\
& \left.f^{(4)}\left(x_{2 i}\right)-\left(x-x_{2 i}\right) f^{(5)}\left(\emptyset_{13_{i} 2 i}\right)\right),
\end{aligned}
$$

From (5) we obtain

$$
f^{(4)}\left(t_{2 i}\right)-f^{(4)}\left(x_{2 i}\right)=\frac{2}{3} h f^{(5)}\left(\phi_{10,2 i}\right) \text { and } S_{n}^{(4)}\left(t_{2 i}\right)-f^{(4)}\left(t_{2 i}\right)=0 .
$$

Substitute this result and (12) in (20), we obtain

$$
\begin{aligned}
& \left|E_{2}\right| \leq 2 h w\left(f^{(5)} ; \frac{1}{m}\right) \\
& E_{4}=4 h^{2} S_{m}^{(6)}\left(t_{2 i}\right) A_{3}\left(\frac{x-2 i h}{2 h}\right),
\end{aligned}
$$

From (15) in lemma 2, we obtain

$\left|E_{4}\right| \leq \frac{5894}{61} h w\left(f^{(5)} ; \frac{1}{m}\right)$

Putting (18)-(23) in (17) we obtain

$$
\left|S_{n}^{(4)}(x)-f^{(4)}(x)\right| \leq \frac{1003064}{8235} h w\left(f^{(5)} ; \frac{1}{m}\right)+4 h\left\|f^{(5)}\right\|_{x}
$$

This proves Theorem 2 for $\mathrm{r}=4$. To prove Theorem 2 for $\mathrm{r}=3$, since $S_{n}^{(3)}(x)-f^{(3)}(x)=\int_{t_{n i}}^{x}\left(S_{n i}^{(4)}(t)-f^{(4)}(t)\right) d t+S_{n}^{m}\left(t_{2 i}\right)-f^{m}\left(t_{2 i}\right)$.

On using (14) and (24) we obtain

$$
\left|S_{n}^{(3)}(x)-f^{(3)}(x)\right| \leq \frac{1346983}{5490} h^{2} w\left(f^{(5)} ; \frac{1}{m}\right)+8 h^{2}\left\|f^{(5)}\right\|_{m}
$$


Which proves Theorem 2 for $r=3$. To prove Theorem 2 for $r=2$, since

$S_{n}^{\omega}(x)-f^{\omega}(x)=\int_{t_{n i}}^{\infty}\left(S_{n}^{(3)}(t)-f^{(3)}(t)\right) d t+S_{n}^{\omega}\left(t_{2 i}\right)-f^{\omega}\left(t_{2 i}\right)$.

Since $S_{n}^{\varpi}\left(t_{2 i}\right)-f^{ \pm \prime}\left(t_{2 i}\right)=0$, and using (25) we obtain

$\left|S_{n}^{\infty}(x)-f^{\infty}(x)\right| \leq \frac{1346983}{2745} h^{3} w\left(f^{(5)} ; \frac{1}{m}\right)+16 h^{3}\left\|f^{(5)}\right\|_{m}$

Which proves Theorem 2 for $\mathrm{r}=2$. To prove Theorem 2 for $\mathrm{r}=1$, since

$S_{n}^{0}(x)-f^{v}(x)=\int_{t_{\mathrm{ni}}}^{x}\left(S_{n}^{(2)}(t)-f^{(2)}(t)\right) d t+S_{n}^{v}\left(t_{2 i}\right)-f^{v}\left(t_{2 i}\right)$.

Since $S_{n}^{\sigma}\left(t_{2 i}\right)-f^{\circ}\left(t_{2 i}\right)=0$, and using (13) and (26), we obtain

$\left|S_{n}^{\sigma}(x)-f^{5}(x)\right| \leq \frac{72745553}{74115} h^{4} w\left(f^{(5)} ; \frac{1}{m}\right)+32 h^{4}\left\|f^{(5)}\right\|_{m}$

This proves Theorem 2 for $r=1$. To prove Theorem 2 for $r=0$, since

$S_{n}(x)-f(x)=\int_{t_{\mathrm{ni}}}^{\infty}\left(S_{n}^{*}(t)-f^{\circ}(t)\right) d t+S_{n}\left(t_{2 i}\right)-f\left(t_{2 i}\right)$,

$S_{n}\left(t_{2 i}\right)-f\left(t_{2 i}\right)=0$, and using (27) we obtain

$\left|S_{n}(x)-f(x)\right| \leq \frac{145491106}{74115} h^{5} w\left(f^{(5)} ; \frac{1}{m}\right)+64 h^{5}\left\|f^{(5)}\right\|_{\infty}$

$\left|S_{n}(x)-f(x)\right| \leq \frac{145491106}{2371680} m^{-5} w\left(f^{(5)} ; \frac{1}{m}\right)+2 m^{-5}\left\|f^{(5)}\right\|_{g x}$

This completes the proof of theorem 2 .

\section{Numerical Results:}

This Section present numerical result to evaluate the maximum error bounds of the spline and it's derivatives which constructed in eq. (3) and also in [4] that showing minimizing error bounds as practice by changes boundary condition and the class of spline. Tables (1-4) in below shows the maximum absolute error between the spline function and it's derivatives which defined in (3) but tables (5-8) shows the maximum absolute error between the spline function and it's derivatives which defined in [4], and also we obtain the really, that is the spline function defined in (3) better to use for interpolating (or approximating) $f(x)$ than the spline which defined in [4].

Problem: See [4] Consider the function $f(x)=x \exp (x)$ and $n=9$.

Table 1. Absolute maximum error for $S_{0}(x)$ and it's derivative.

\begin{tabular}{|c|c|c|c|}
\hline$x$ & $\left\|S_{0}(x)-f(x)\right\|$ & $\left\|S_{0}^{*}(x)-f^{*}(x)\right\|$ & $\left\|S_{0}^{*}(x)-f^{*}(x)\right\|$ \\
\hline 0 & 0 & $0.040999999950664^{*} 10^{-6}$ & $0.114299999998124 * 10^{-5}$ \\
\hline 0.125 & $0.71370023779771 * 10^{-9}$ & $0.005220108567627^{*} 10^{-6}$ & $0.082786173249971 * 10^{-5}$ \\
\hline 0.25 & $0.414490441968951 * 10^{-9}$ & $0.100777041822653^{*} 10^{-6}$ & $0.400793695698454 * 10^{-5}$ \\
\hline
\end{tabular}

\begin{tabular}{|c|c|c|c|}
\hline$x$ & $\left\|S_{0}^{(m)}(x)-f^{\prime \prime \prime}(x)\right\|$ & $\left\|S_{0}^{(4)}(x)-f^{(4)}(x)\right\|$ & $\left\|S_{0}^{(5)}(x)-f^{(5)}(x)\right\|$ \\
\hline 0 & 0 & $0.075808000000 * 10^{-3}$ & $2.2236800000000 * 10^{-2}$ \\
\hline 0.125 & $0.106238650823265 * 10^{-4}$ & $0.683054349341 * 10^{-3}$ & $1.4790678032516^{*} 10^{-2}$ \\
\hline 0.25 & $0.454875148401257 * 10^{-4}$ & $1.639703922900 * 10^{-3}$ & $7.4543637610642 * 10^{-2}$ \\
\hline
\end{tabular}


Table 2. Absolute maximum error for $S_{1}(x)$ and it's derivative.

\begin{tabular}{|c|c|c|c|}
\hline$x$ & $\left\|S_{1}(x)-f(x)\right\|$ & $\left\|S_{1}^{*}(x)-f^{*}(x)\right\|$ & $\left\|S_{1}^{*}(x)-f^{*}(x)\right\|$ \\
\hline 0.25 & $0.058984558792829 * 10^{-6}$ & $0.045201727649768^{*} 10^{-5}$ & $0.415491351946074 * 10^{-5}$ \\
\hline 0.375 & $0.148453526804815^{*} 10^{-6}$ & $0.089293177252259 * 10^{-5}$ & $0.080846292460635^{*} 10^{-5}$ \\
\hline 0.5 & $0.263634310826433 * 10^{-6}$ & $0.100019980786570^{*} 10^{-5}$ & $0.227012467979293 * 10^{-5}$ \\
\hline
\end{tabular}

\begin{tabular}{|c|c|c|c|}
\hline$x$ & $\left\|S_{1}^{\left({ }^{\prime \prime}\right.}(x)-f^{\prime \prime \prime}(x)\right\|$ & $\left\|S_{1}^{(4)}(x)-f^{(4)}(x)\right\|$ & $\left\|S_{1}^{(5)}(x)-f^{(5)}(x)\right\|$ \\
\hline 0.25 & $0.506538898408238^{*} 10^{-4}$ & $1.774618422900 * 10^{-3}$ & $0.5620257610643^{*} 10^{-2}$ \\
\hline 0.375 & $0.424498833044851 * 10^{-4}$ & $0.863774170369 * 10^{-3}$ & $2.1792936427169 * 10^{-2}$ \\
\hline 0.5 & $0.134974504488738^{*} 10^{-4}$ & $3.319368150576^{*} 10^{-3}$ & $11.8736588850705^{*} 10^{-2}$ \\
\hline
\end{tabular}

Table 3. Absolute maximum error for $S_{2}(x)$ and it's derivative.

\begin{tabular}{|c|c|c|c|}
\hline$x$ & $\left\|S_{2}(x)-f(x)\right\|$ & $\left\|S_{2}^{*}(x)-f^{*}(x)\right\|$ & $\left\|S_{2}(x)-f^{*}(x)\right\|$ \\
\hline 0.5 & $0.187503756410301 * 10^{-5}$ & $0.157623001921792 * 10^{-4}$ & $0.098249250320759 * 10^{-3}$ \\
\hline 0.625 & $0.470748411296462 * 10^{-5}$ & $0.303969272148485 * 10^{-4}$ & $0.137164324037542 * 10^{-3}$ \\
\hline 0.75 & $0.964247415469188 * 10^{-5}$ & $0.488555956184022 * 10^{-4}$ & $0.152300216105239 * 10^{-3}$ \\
\hline
\end{tabular}

\begin{tabular}{|c|c|c|c|}
\hline$x$ & $\left\|S_{2}^{\text {(I) }}(x)-f^{\text {(I) }}(x)\right\|$ & $\left\|S_{2}^{(4)}(x)-f^{(4)}(x)\right\|$ & $\left\|S_{2}^{(5)}(x)-f^{(5)}(x)\right\|$ \\
\hline 0.5 & $0.230847450448657 * 10^{-3}$ & $0.2480718150577 * 10^{-2}$ & $0.5341011149294 * 10^{-2}$ \\
\hline 0.625 & $0.282941004305926^{*} 10^{-3}$ & $0.2312009375972 * 10^{-2}$ & $3.6761489443750 * 10^{-2}$ \\
\hline 0.75 & $0.050649797530333 * 10^{-3}$ & $0.2573828910204 * 10^{-2}$ & $15.4768095522879 * 10^{-2}$ \\
\hline
\end{tabular}

Table 4. Absolute maximum error for $S_{3}(x)$ and it's derivative.

\begin{tabular}{|c|c|c|c|}
\hline$x$ & $\left\|S_{3}(x)-f(x)\right\|$ & $\left\|S_{3}^{s}(x)-f^{s}(x)\right\|$ & $\left\|S_{3}^{s \prime}(x)-f^{\prime \prime}(x)\right\|$ \\
\hline 0.75 & $0.93110907681604 * 10^{-4}$ & $0.317171630944024 * 10^{-3}$ & $0.581399940144500 * 10^{-3}$ \\
\hline 0.875 & $1.37507711833074 * 10^{-4}$ & $0.394877268722116^{*} 10^{-3}$ & $0.664878086780618^{*} 10^{-3}$ \\
\hline 1 & $1.92378540954330 * 10^{-4}$ & $0.485963081908736^{*} 10^{-3}$ & $0.802474622862981 * 10^{-3}$ \\
\hline
\end{tabular}

\begin{tabular}{|c|c|c|c|}
\hline$x$ & $\left\|S_{3}^{m \prime \prime}(x)-f^{\prime \prime \prime}(x)\right\|$ & $\left\|S_{3}^{(4)}(x)-f^{(4)}(x)\right\|$ & $\left\|S_{3}^{(5)}(x)-f^{(5)}(x)\right\|$ \\
\hline 0.75 & $0.665112702469 * 10^{-3}$ & $0.1712318910204 * 10^{-2}$ & $0.15153104477122^{*} 10^{-1}$ \\
\hline 0.875 & $0.815543377493 * 10^{-3}$ & $0.4674651910397 * 10^{-2}$ & $0.41167647943299 * 10^{-1}$ \\
\hline 1 & $1.224626163818 * 10^{-3}$ & $0.3731382295229 * 10^{-2}$ & $2.28474170754271 * 10^{-1}$ \\
\hline
\end{tabular}

Table 5. Absolute maximum error for $R_{0}(x)$ and it's derivative.

\begin{tabular}{|c|c|c|c|}
\hline$x$ & $\left\|R_{0}(x)-f(x)\right\|$ & $\left\|R_{0}^{\prime}(x)-f^{\prime}(x)\right\|$ & $\left\|R_{0}^{\prime \prime}(x)-f^{\prime \prime}(x)\right\|$ \\
\hline 0 & 0 & 0 & $0.341182352500000 * 10^{+2}$ \\
\hline 0.125 & 0.130721478797250 & 2.644457893742201 & $0.390373071046159 * 10^{+2}$ \\
\hline 0.25 & 2.034423779011657 & 45.512962248671549 & $9.000514326405780 * 10^{+2}$ \\
\hline
\end{tabular}

\begin{tabular}{|c|c|c|c|}
\hline$x$ & $\left\|R_{0}^{m \prime \prime}(x)-f^{m \prime \prime}(x)\right\|$ & $\left\|R_{0}^{(4)}(x)-f^{(4)}(x)\right\|$ & $\left\|R_{0}^{(5)}(x)-f^{(5)}(x)\right\|$ \\
\hline 0 & $0.348774214000000 * 10^{+4}$ & $1.173445947000000 * 10^{+5}$ & $1.971389178000000 * 10^{+6}$ \\
\hline 0.125 & $0.141199926749042 * 10^{+4}$ & $0.616595361563811 * 10^{+5}$ & $0.892676896114178^{*} 10^{+6}$ \\
\hline
\end{tabular}




\begin{tabular}{|l|l|l|l|}
\hline 0.25 & $1.328433273864577 * 10^{+4}$ & $1.058246240669791 * 10^{+5}$ & $0.186035512133438 * 10^{+6}$ \\
\hline
\end{tabular}

Table 6. Absolute maximum error for $R_{1}(x)$ and it's derivative.

\begin{tabular}{|c|c|c|c|}
\hline$x$ & $\left\|R_{1}(x)-f(x)\right\|$ & $\left\|R_{1}^{s}(x)-f^{s}(x)\right\|$ & $\left\|R_{1}^{v \prime}(x)-f^{v \prime}(x)\right\|$ \\
\hline 0.25 & $0.603113531229562 * 10^{+3}$ & $0.496103988141148 * 10^{+4}$ & $2.188102389625005^{*} 10^{+4}$ \\
\hline 0.375 & $1.396039284183429 * 10^{+3}$ & $0.775899877302860 * 10^{+4}$ & $2.326997000275429 * 10^{+4}$ \\
\hline 0.5 & $2.551302063985349 * 10^{+3}$ & $1.075349478740606^{*} 10^{+4}$ & $2.478581697817677 * 10^{+4}$ \\
\hline
\end{tabular}

\begin{tabular}{|c|c|c|c|}
\hline$x$ & $\left\|R_{1}^{\prime \prime \prime}(x)-f^{\prime \prime \prime}(x)\right\|$ & $\left\|R_{1}^{(4)}(x)-f^{(4)}(x)\right\|$ & $\left\|R_{1}^{(5)}(x)-f^{(5)}(x)\right\|$ \\
\hline 0.25 & $0.778891494864570 * 10^{+4}$ & $6.824478543580213 * 10^{+5}$ & $1.191572722136656^{*} 10^{+7}$ \\
\hline 0.375 & $1.353758044368026 * 10^{+4}$ & $1.083268737250599 * 10^{+5}$ & $0.073666840817114 * 10^{+7}$ \\
\hline 0.5 & $2.335363227444772 * 10^{+4}$ & $4.982807602457182 * 10^{+5}$ & $1.044239057296699 * 10^{+7}$ \\
\hline
\end{tabular}

Table 7. Absolute maximum error for $R_{2}(x)$ and it's derivative.

\begin{tabular}{|c|c|c|c|}
\hline$x$ & $\left\|R_{2}(x)-f(x)\right\|$ & $\left\|R_{2}^{\prime}(x)-f^{\prime}(x)\right\|$ & $\left\|R_{2}^{\prime \prime}(x)-f^{\prime \prime}(x)\right\|$ \\
\hline 0.5 & $0.830464555149896^{*} 10^{+5}$ & $3.296993905180940 * 10^{+5}$ & $7.268974788093231 * 10^{+5}$ \\
\hline 0.625 & $1.300877868285046 * 10^{+5}$ & $4.241133823374287 * 10^{+5}$ & $7.827124902395661 * 10^{+5}$ \\
\hline 0.75 & $1.893623383824973 * 10^{+5}$ & $5.254443003390334 * 10^{+5}$ & $8.382096940382363 * 10^{+5}$ \\
\hline
\end{tabular}

\begin{tabular}{|c|c|c|c|}
\hline$x$ & $\left\|R_{2}^{m \prime \prime}(x)-f^{\prime \prime \prime}(x)\right\|$ & $\left\|R_{2}^{(4)}(x)-f^{(4)}(x)\right\|$ & $\left\|R_{2}^{(5)}(x)-f^{(5)}(x)\right\|$ \\
\hline 0.5 & $4.964680029755498^{*} 10^{+5}$ & $1.803210009245722 * 10^{+6}$ & $3.147259853203301 * 10^{+7}$ \\
\hline 0.625 & $4.401176946787137 * 10^{+5}$ & $0.286975244362445 * 10^{+6}$ & $0.197036549111649 * 10^{+7}$ \\
\hline 0.75 & $4.145543461874368 * 10^{+5}$ & $1.310618645750083 * 10^{+6}$ & $2.753186777275012 * 10^{+7}$ \\
\hline
\end{tabular}

Table 8. Absolute maximum error for $R_{3}(x)$ and it's derivative.

\begin{tabular}{|c|c|c|c|}
\hline$x$ & $\left\|R_{3}(x)-f(x)\right\|$ & $\left\|R_{3}^{\prime}(x)-f^{\prime}(x)\right\|$ & $\left\|R_{3}^{\prime \prime}(x)-f^{\prime \prime}(x)\right\|$ \\
\hline 0.75 & $5.434462147228640 * 10^{+5}$ & $0.631910510663058^{*} 10^{+6}$ & $2.678862080328076^{*} 10^{+6}$ \\
\hline 0.875 & $4.406875618723612 * 10^{+5}$ & $1.036309377388700 * 10^{+6}$ & $3.836308395394652 * 10^{+6}$ \\
\hline 1 & $2.775914504818287 * 10^{+5}$ & $1.603572860436344^{*} 10^{+6}$ & $5.292958127154512 * 10^{+6}$ \\
\hline
\end{tabular}

\begin{tabular}{|c|c|c|c|}
\hline$x$ & $\left\|R_{3}^{n \prime \prime}(x)-f^{\prime \prime \prime}(x)\right\|$ & $\left\|R_{3}^{(4)}(x)-f^{(4)}(x)\right\|$ & $\left\|R_{3}^{(5)}(x)-f^{(5)}(x)\right\|$ \\
\hline 0.75 & $0.825180448593744 * 10^{+7}$ & $1.370223304424992 * 10^{+7}$ & $6.904428095224993 * 10^{+7}$ \\
\hline 0.875 & $1.039028672322542 * 10^{+7}$ & $1.960384008048292 * 10^{+7}$ & $2.538143141910770 * 10^{+7}$ \\
\hline 1 & $1.292535382687269 * 10^{+7}$ & $2.004759090859086^{*} 10^{+7}$ & $1.828141840969094 * 10^{+7}$ \\
\hline
\end{tabular}

\section{Conclusions}

In this paper, we conclude that the changes of the boundary conditions and the class of spline functions together affect on minimizing error bounds in the subject of lacunary interpolation by spline functions theoretically and practically, also we can use in the further the same idea for different lacunary data for example $(0,1,3),(0,3,5) \ldots$ etc. 


\section{REFERENCES}

[1] Ahlberg, J.H., E.N. Nilson and J.L Walsh, (1967), The theory of splines and their applications, Academic Press, New York and London.

[2] Birkoff, G.D., (1906), General mean value and remainder theorem with application to mechanical differentiation and integration, Trans. Amer. Math. Soc., 17:107-136.

[3] Jwamer, K.H., (2007), Minimizing error bounds in $(0,2,3)$ lacunary interpolation by sixtic spline function, Journal of Mathematics and Statistics, USA, 3(4), pp.249-256 .

[4] Mishra, R.S. and Muthur, K.K., (1980), Lacunary interpolation by spline $(0,2,3)$ and $(0,2,4)$ cases, Acta Math. Acad. Sci. Hungar., 36(3-4), pp.251-260.

[5] Richard L. Burden and J. Douglas Faires, (2002), Numerical Analysis, $7^{\text {th }}$ edition, Printed in USA.

[6] Saeed, R.K. and Jwamer, K.H., (2005), Minimizing error bounds in Lacunary interpolation by spline function, $(0,1,4)$ case. Journal of Al. Nahrain University, 8 (2), pp.114-119.

[7] Schoenberg, I.J., (1968), On Ahlberg-Nilson extent ion of spline interpolation: the g-spline and their Optimal properties, J. Math. Anal. Appl., (2), pp.207-231. 